\title{
Whither the Price of Bitcoin?
}

\section{David Andolfatto, Senior Vice President and Economist}

Andrew Spewak, Senior Research Associate

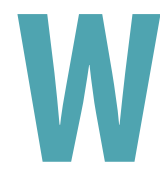

hat are the long-run prospects of Bitcoin as an investment? The bullish case is that Bitcoin will appreciate indefinitely due to its capped supply and an ever-growing demand. The bearish case is that Bitcoin's price will fall to zero, as it's an intrinsically worthless asset. We think the future price path is more likely to remain bounded between these two extremes.

Consider first the bullish case for Bitcoin. We think this idea is too optimistic even if one grants that its supply is fixed and its demand is likely to grow. ${ }^{1}$ The U.S. dollar price of Bitcoin will also depend on how its exchange rate relative to other cryptocurrencies evolves over time in the face of an ever-expanding supply of alternative cryptocurrencies, which we refer to as Altcoin. ${ }^{2}$

The bullish case assumes that the nominal exchange rate between Bitcoin vis-à-vis other cryptocurrencies will adjust in proportion to their relative supplies. That is, Bitcoin is expected to appreciate relative to its competitors or, equivalently, its market-capitalization share will stay constant over time. But must this necessarily be the case?

Consider the following thought experiment. A restaurant selling meals for $\$ 10$ will happily accept payment in the form of one Hamilton bill (\$10) or two Lincoln bills (\$5). That is, the nominal exchange rate between Hamilton and Lincoln bills is 2:1. Now, suppose that the supply of Lincoln bills is increased but the supply of Hamilton bills remains the same. The exchange rate remains unaffected, so the total nominal money supply is increased. If the demand for money remains unchanged, then the price level must rise. Consequently, neither a single Hamilton bill nor two Lincoln bills is sufficient to purchase a meal, which now costs more than $\$ 10$. That is, the increase in the supply of Lincoln bills has led to a decline in the purchasing power of both Lincoln bills and Hamilton bills, even though the supply of Hamilton bills has remained fixed. Might an expansion in the supply of Altcoin have a similar depressing effect on the price of Bitcoin?

The analogy is not perfect, because the exchange rates between competing cryptocurrencies are not fixed in the way the exchange rate is for Hamilton and Lincoln bills. What would determine the market exchange rate between Hamilton and Lincoln bills if the numbers on these bills were erased? According to economic theory, any exchange rate is possible-including an exchange rate that does not

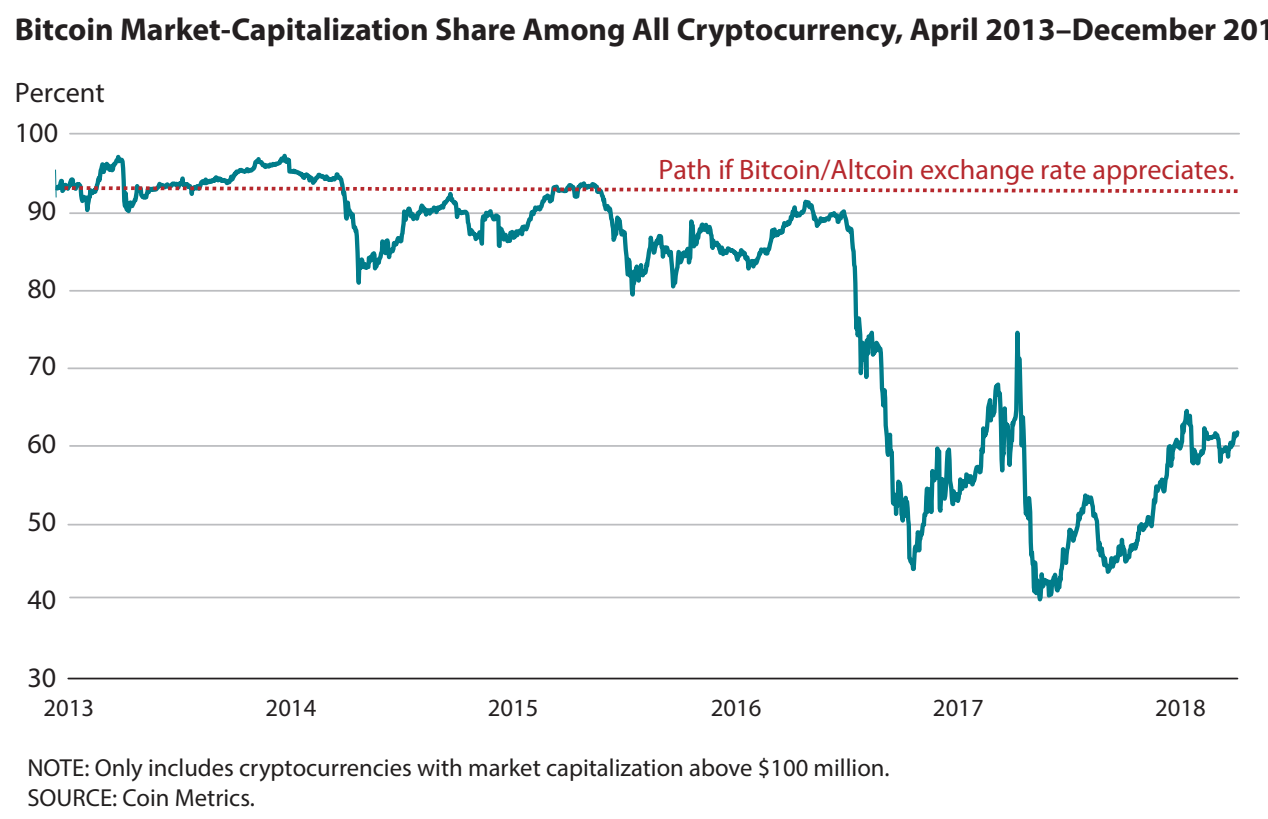


vary with the relative supplies of Hamilton and Lincoln bills. ${ }^{3}$ The intuition for this result is that Hamilton and Lincoln bills are intrinsically worthless bits of paper. There are no fundamental economic factors determining the exchange rate between two intrinsically worthless objects. Something similar holds true for Bitcoin and Altcoin.

Thus, while it is possible that an increase in the supply of Altcoin results in Bitcoin exchange-rate appreciation with respect to Altcoin, this need not be the case. And if not, then an ever-expanding supply of Altcoin would keep the price of Bitcoin (and, by extension, its market capitalization) depressed relative to where it would have otherwise been.

\section{The total value of Bitcoin has declined as a share of all cryptocurrencies.}

The figure suggests exactly this effect. The total value of Bitcoin has declined as a share of all cryptocurrencies, as its value with respect to other cryptocurrencies shows no particular trend.

Consider now the bearish case for Bitcoin. This outlook is based on the view that Bitcoin has no fundamental value and that sooner or later the market will recognize this fact. In our view, one can accept that Bitcoin trades above its fundamental value without claiming that its fundamental value is zero. In fact, many securities trade above what might be considered their fundamental value. Gold, for example, trades above its value as measured by its industrial applications. The U.S. dollar trades above its fundamental value in discharging U.S. tax obligations. The premium some people are willing to pay for gold and the U.S. dollar reflects the value these objects possess as exchange media. The market value of these objects would decline, but not fall to zero, should this premium suddenly vanish.

What is the source of the fundamental value of Bitcoin? Think about it this way. At its core, Bitcoin is a database management system. Database management systems can have a fundamental value if they are tailored to meet the needs of a given constituency. Bitcoin offers people a money storage and transfer system with two key properties: (i) permissionless access and (ii) decentralized database management. The first property means that no one can prevent a user from sending any amount of Bitcoin from one account to another. The second property means that the protocol does not depend on the existence of a delegated authority to manage accounts and transfer funds. The fundamental demand for Bitcoin derives from the fact that there are at least some people who value these features. ${ }^{4}$ This fundamental demand provides a non-zero lower bound on the price of Bitcoin. ${ }^{5}$

To sum up, economic theory predicts that the price dynamic of an unbacked asset is likely to be highly volatile and inherently unforecastable. ${ }^{6}$ While Bitcoin's price is not likely to fall to zero, the prospect of a flood of Altcoin competing with Bitcoin in the wealth portfolios of investors is likely to place significant downward pressure on the purchasing power of all cryptocurrencies, including Bitcoin.

\section{Notes}

1 The supply of Bitcoin in the orthodox version of Bitcoin is capped at 21 million units. Any attempt to modify this cap is likely to result in heterodox versions of Bitcoin (so-called "hard forks") rather than in any reformation of the orthodoxy.

2 The supply of Altcoin is potentially infinite because Bitcoin is an opensource software that can be costlessly replicated.

3 See Kareken, John and Wallace, Neil. "On the Indeterminacy of Equilibrium Exchange Rates." Quarterly Journal of Economics, May 1981, 96(2), pp. 207-22.

4 There may also be fundamental demand stemming from the fact that some people value Bitcoin because of its well-established network of users (called the "network effect") or because it was the first cryptocurrency (the "first-mover advantage").

5 Such a lower bound is modeled in King, Robert G.; Wallace, Neil and Weber, Warren E. "Nonfundamental Uncertainty and Exchange Rates." Journal of International Economics, February 1992, 32(1-2), pp. 83-108.

${ }^{6}$ See Manuelli, Rodolfo E. and Peck, James. "Exchange Rate Volatility in an Equilibrium Asset Pricing Model." International Economic Review, August 1990, 31(3), pp. 559-74. 